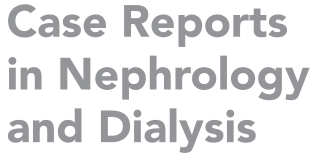

Case Reports and Dialysis

\title{
An Overlooked Link between IgA Nephropathy and Lithium Toxicity: A Case Report
}

\author{
Sushil K. Mehandru ${ }^{a}$ Supreet Kaur ${ }^{a} \quad$ Aisha Ghias ${ }^{a} \quad$ Mohamed Bakr $^{a}$ \\ Arif Asifa $^{a}$ Tushar J. Vachharajani ${ }^{b}$
}

aDivision of Nephrology \& Hypertension, Department of Medicine, Jersey Shore University Medical Center, Hackensack Meridian School of Medicine, Neptune, NJ, USA; bepartment of Nephrology and Hypertension, Glickman Urological \& Kidney Institute, Cleveland Clinic, Cleveland Clinic Lerner College of Medicine of Case Western Reserve University, Cleveland, $\mathrm{OH}$, USA

\section{Keywords}

Nephropathy $\cdot \lg A \cdot$ Lithium $\cdot$ Sodium lithium counter-transporter $\cdot$ Bipolar disorder

\begin{abstract}
Lithium is one of the first-line agents for treating bipolar disorder. Although this agent is highly effective in treating mood disorders, renal toxicity is a frequent side effect. Lithium metabolism is affected by sodium-lithium counter-transporter (SLC-T) in erythrocytes. The high activity of SLC-T can result in decreased urinary lithium clearance and may lead to accumulation of lithium in the distal renal tubular cells, causing lithium toxicity. SLC-T is a genetic marker in primary hypertension (HTN), HTN in pregnancy, diabetic nephropathy, and IgA nephropathy $(\lg \mathrm{A}-\mathrm{N})$ with HTN. Patients with $\lg \mathrm{A}-\mathrm{N}$ have been reported to have enhanced SLC-T activity and are likely to have considerably lower renal fractional clearance of lithium. Therefore, patients taking lithium for bipolar disorder with coexisting $\lg \mathrm{A}-\mathrm{N}$ can have severe lithium-induced nephropathy and nephrotoxicity even at therapeutic serum levels. Serum lithium levels reflect only extracellular lithium concentration. However, lithium exerts its effects once it has moved to the intracellular compartment. This phenomenon illustrates the reason why patients with significantly elevated serum levels might be asymptomatic. Creatinine clearance is inversely related to the duration of lithium therapy. The degree of interstitial fibrosis on renal biopsy has been known to be associated with the duration of lithium therapy and cumulative dose. We present a case with a past medical history of bipolar disorder treated with lithium for almost 20 years. His family history was significant for HTN. The patient was diagnosed with renal insufficiency of unknown causes, for which he underwent renal biopsy. The renal biopsy showed a typical lithium-induced tubulointerstitial nephritis and a
\end{abstract}


coincidental finding of $\lg \mathrm{A}-\mathrm{N}$. We suspect a high activity of SLC-T seen in $\lg \mathrm{A}-\mathrm{N}$, and the adverse effects of lithium on SLC-T activity might cause reduction of urinary lithium clearance and accumulation of lithium in distal renal tubular cells, contributing to nephrotoxicity. There is a lack of the literature on the coexistence of IgA-N and lithium nephrotoxicity. We recommend in patients with concomitant IgA-N, taking lithium, more frequent monitoring of renal functions, and dose adjustments may reduce the risk of lithium-induced nephrotoxicity.

(C) 2021 The Author(s).

Published by S. Karger AG, Basel

\section{Introduction}

In the USA, mood disorders such as bipolar I and bipolar II afflict roughly $2.8 \%$ of the population annually [1]. Mood disorders affect approximately 46 million people worldwide [2]. Lithium is one of the first-line agents used for treating bipolar disorders and is highly effective. Renal toxicity is a common side effect of lithium. Lithium (Li) transport in the kidneys is similar to that of sodium (Na). Roughly $60 \%$ of $\mathrm{Li}$ is reabsorbed in the proximal convoluted tubule. Dehydration, NSAIDS, ACEI, and renal insufficiency can increase lithium reabsorption, resulting in lithium toxicity [3]. Therefore, lithium treatment warrants routine monitoring of lithium and serum creatinine levels.

Interestingly, serum lithium levels reflect only extracellular lithium concentration. However, lithium exerts its effects once it has moved to its intracellular compartment. This phenomenon illustrates the reason why patients with significantly elevated serum levels might be asymptomatic [4]. Creatinine clearance is inversely related to the duration of lithium therapy. The degree of interstitial fibrosis on renal biopsy has also been known to be associated with lithium duration and cumulative dose [5].

Roughly $40 \%$ of the remaining Li reabsorption occurs at the loop of Henle and the distal nephron. Various transport channels, such as ouabain-sensitive sodium/potassium ATPase channel, amiloride-sensitive sodium/hydrogen $(\mathrm{Na} / \mathrm{H})$ exchange channels, furosemidesensitive sodium-potassium-chloride (Na-K-2Cl) channel, and sodium-dependent cotransporters have been postulated for the transport of lithium into the cells. Still, the same channel or transporter for Li efflux is unknown [6]. One proposed transporter is sodiumlithium counter-transporter (SLC-T), unanimously found in erythrocytes but might also be present in the kidneys. SLC-T is ouabain-insensitive and catalyzes as an exchange for sodium (or lithium) against lithium (or sodium), where it has roughly $20 \%$ more affinity for lithium than sodium [6]. It seems, though, that the electrolyte concentration gradient drives the sodium and lithium movement across SLC-T in erythrocytes. Since sodium concentration is high outside the cell compared to inside the cell, transport down the $\mathrm{Na}$ gradient from outside the cell and transport down the lithium gradient inside the cell might be the driving force for efflux for lithium [6]. The exact physiology as to what catalyzes this exchange has yet to be determined.

SLC-T is now generally accepted as an inheritable marker in primary hypertension (HTN) [5]. SLC-T is presumably a genetic marker in primary HTN, HTN in pregnancy, diabetic nephropathy, and IgA nephropathy (IgA-N) with HTN. Other common diseases, such as type 1 diabetes mellitus with nephropathy and retinopathy, pregnancy, HTN in pregnancy, hyperlipidemia, and type II diabetes with nephropathy, all tend to have high SLC-T expressions [7, 8]. SLC-T expression is also elevated in IgA-N, especially in IgA-N with HTN [7]. Almost 50\% of patients affected by IgA-N develop HTN and subsequently develop renal failure due to tubulointerstitial damage [9].

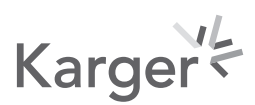


A small retrospective study by Kontessis et al. [10] showed that a higher SLC-T rate correlated with the faster deterioration of renal function in patients with IgA-N and HTN, regardless of being on antihypertensive treatments. Having high SLC-T expression as a marker in many disease processes, such as HTN and diabetic nephropathy, including IgA-N with HTN, indicates the genetic susceptibility to these diseases, leading to decline of renal function. The genetic link of SLC-T to HTN further supported that its activity is controlled by the SLC20A1 gene [11].

One of the earliest pioneers, Dr. Weder [12], studied a possible link between SLC-T expression and sodium reabsorption in hypertensive patients. He measured urinary lithium clearance as a measure of sodium reabsorption in the proximal convoluted tubule. He studied 14 subjects with untreated HTN and 31 normotensive serving as controls. Volunteers were all white men between 18 and 50 years of age and had no other primary medical history, except mild HTN in the study group. All subjects were within $25 \%$ of their ideal body weight. HTN was defined as having a diastolic blood pressure $>90 \mathrm{~mm} \mathrm{Hg}$. The exclusion criteria were any antihypertensive, and aspirin or acetaminophen use within the past 2 weeks. All subjects took $600 \mathrm{mg}$ of oral lithium carbonate with water at 10:00 p.m., fasted overnight, and began $6 \mathrm{~h}$ urine collection. Also, the subjects were fasting, except for the intake of water throughout the collection of urine for lithium. A single plasma sample at the midpoint of urine collection was deemed adequate for calculating the clearance of lithium by the kidneys [12]. The activity of erythrocyte SLC-T was measured by Canessa et al.'s [13] method.

Subjects with a negative family history of HTN, either normotensive or hypertensive, had a higher fractional clearance of lithium [12]. These findings suggest that fractional lithium clearance has an inverse relationship with high SLC-T activity, mainly seen in hypertensive patients. Having low fractional lithium clearance among subjects with a positive family history of HTN makes one suspect that high SLC-T activity precedes development of HTN. Our brief review of the literature shows that SLC-T activity is high among those with HTN, a family history of HTN, and IgA-N with HTN. We speculate that lithium use among patients with high SLC - T activity, as seen in HTN, IgA-N with a family history of HTN, might decrease urinary lithium fractional clearance. Ultimately, a decreased lithium clearance can result in lithium toxicity and/or lithium-induced tubulointerstitial nephropathy.

\section{Case Study}

A 54-year-old Caucasian man with a history of bipolar disorder was referred to the nephrologist by his primary care physician for renal insufficiency. The patient had been on lithium therapy for almost 20 years. The patient had no known history of HTN but has a significant family history of HTN. Renal ultrasound showed a right kidney size of $10.0 \mathrm{~cm}$ and the left kidney size of $15.0 \mathrm{~cm}$. The patient is 5 feet and 11 inches tall. Ultrasound also revealed increased echogenicity and multiple renal cysts bilaterally. Urine analysis revealed trace blood, 15-20 WBC/HPF, and 3-5 RBCs/HPF, negative for protein. Serum levels of C3 and C4 were normal. Creatinine was at $1.8 \mathrm{mg} / \mathrm{dL}$ and GFR $43 \mathrm{cc} / \mathrm{min}$.

A renal biopsy was performed to determine the etiology of renal insufficiency. The pathology report of the renal biopsy showed (read by Dr. Samih Nasr, MD of Columbia University):

Upon microscopic examination and staining, the sections with H\&E, PAS, trichrome, and JMS revealed one globally sclerotic glomerulus. One glomerulus revealed a mild segmental hypercellularity, whereas the remaining glomeruli were unremarkable. Moderate tubular atrophy and interstitial fibrosis with distal tubular cysts were found, consistent with chronic lithium toxicity. Significant findings upon immunofluorescence revealed 2+ granular global

\section{Karger'}



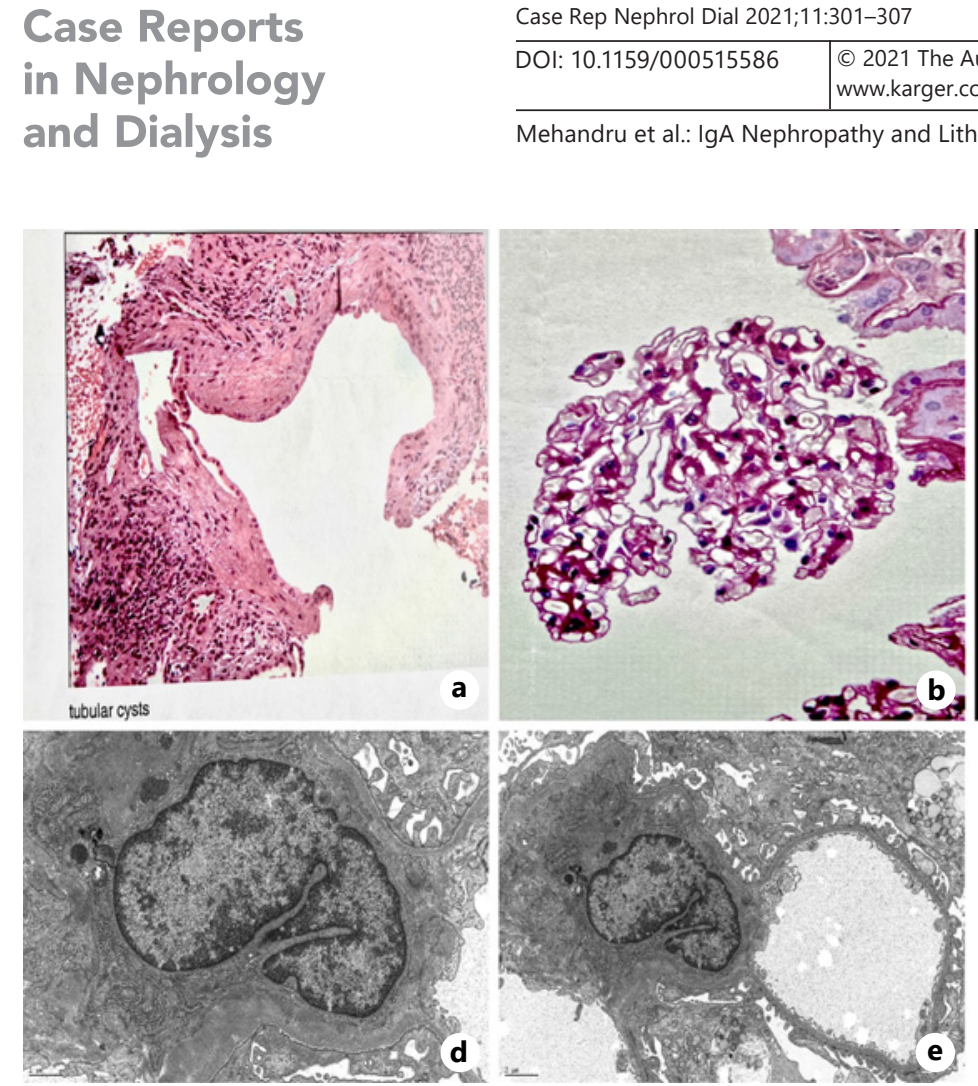

\begin{tabular}{l} 
Case Rep Nephrol Dial 2021;11:301-307 \\
\hline DOI: 10.1159/000515586
\end{tabular} \begin{tabular}{l}
$\begin{array}{l}\text { c 2021 The Author(s). Published by S. Karger AG, Basel } \\
\text { www.karger.com/cnd }\end{array}$ \\
\hline
\end{tabular}

Mehandru et al.: IgA Nephropathy and Lithium Toxicity

Fig. 1. a Moderate tubular atrophy and interstitial fibrosis with distal tubular cyst. b Mild mesangial hypercellularity. c Immunofluorescence demonstrating 2+ mesangial staining for IgA electron microscopy. d, e mild segmental mesangial hypercellularity and para-mesangial electron-dense deposits.

mesangial positivity for IgA with negative IgG, negative IgM, negative C3 and C1, trace kappa, and 1-2+ lambda, supporting the diagnosis of IgA-N.

On electron microscopic examination, mild segmental mesangial hypercellularity was found (Fig. 1d, e). Several small segmental para-mesangial electron-dense deposits were identified. Glomerular peripheral capillaries were present. No endothelial tubuloreticular inclusions are seen. Podocytes display mild foot process effacement involving approximately $10 \%$ of the total peripheral capillary surface area. Tubular cells contain electron-dense protein reabsorption droplets. There was mild arteriosclerosis with hyalinosis.

It is important to note that there was no reason to suspect IgA-N before biopsy. Throughout the span of 2 years post-biopsy and after discontinuing the use of lithium, the patient's renal functions remained stable with a GFR in the range of 30-36 cc/min, Cr range of 1.8-2.38 mg/ $\mathrm{dL}$, and BUN ranging from 8 to $21 \mathrm{mg} / \mathrm{dL}$.

\section{Discussion}

Lithium accumulates in the distal tubular cells of the kidneys at concentrations 10-20 times higher than those in the serum. Chronic treatment with lithium commonly produces a defect in the concentrating ability of the kidney due to inhibition of generation of cyclic adenosine monophosphate by antidiuretic hormone at the distal tubule, with lithium probably acting via adenylate cyclase and possibly also at a point distal to the generation of cyclic adenosine monophosphate [4]. About $30-90 \%$ of patients show lowered maximum urine osmolality [14].

Our case brings many essential questions to the surface regarding SLC-T expression as a marker and a transporter involved in lithium metabolism and clearance. As mentioned before, 
Table 1. Classic findings upon renal biopsy of lithium nephrotoxicity [12] and Ig-N [13]

Pathology of lithium nephrotoxicity and IgA-N (in adults)

\begin{tabular}{ll}
\hline Lithium nephrotoxicity (chronic interstitial nephritis) & IgA-N \\
\hline Interstitial fibrosis & $\begin{array}{l}\text { Mesangial deposition of IgA (also along } \\
\text { glomerular capillary wall) }\end{array}$ \\
$\begin{array}{l}\text { Tubular lesions (i.e., dilatation of tubules in } \\
\text { the distal segments and collecting ducts) }\end{array}$ & $\begin{array}{l}\text { Segmental necrosis with or without crescent } \\
\text { formation } \\
\text { Tubular cysts }\end{array}$ \\
$\begin{array}{l}\text { Glomerulosclerosis (late manifestation) } \\
\text { (late manifestation) }\end{array}$ & Mesangial hypercellularity \\
& Tubular atrophy \\
& Interstitial fibrosis \\
\hline
\end{tabular}

abnormal SLC-T expression, as a marker, is seen in primary HTN, IgA-N with HTN, and IgA-N with a significant family history of HTN $[11,15,16]$. Possibly, as an active transporter, SLC-T not only affects lithium efflux from the red cells but also chronic lithium use affects the kinetics of SLC-T $[17,18]$. A study by Ehrlich and Diamond [18] showed that lithium counter-transport efflux could be inhibited by $50 \%$ upon chronic administration of lithium in manic patients. This efflux stays inhibited as long as all carriers are saturated.

Our patient was susceptible to nephropathy not only for being on chronic lithium therapy but also for having a family history of HTN. Both risk factors collectively lead to likely dynamic changes in SLC-T kinetics and low urinary lithium clearance. Sequentially, lithium accumulation caused renal damage as seen on biopsy: tubular cysts, tubular dilatation, and interstitial fibrosis (see Fig. 1a-c). This pathology is consistent with biopsy findings reported by Markowitz et al. [19] that often seen in chronic lithium use with progressive glomerular and tubulointerstitial nephropathy [13].

Studies by Boero et al. $[16,20]$ reported a correlation between increased SLC-T activity in subjects with IgA-N and IgA-N with HTN. They found $>50 \%$ of IgA-N patients develop HTN [16]. Genetic factors and variability in SLC-T kinetics might already be present among IgA-N patients before presenting with IgA-N or HTN development $[16,17]$. Our patient had a family history of HTN and had an incidental finding of IgA-N on renal biopsy. His renal pathology report showed mesangial proliferation and para-mesangial electron-dense deposits, consistent with a mild course of IgA-N (see Fig. 1b, c). Since our patient had a family history of HTN, IgA-N, and chronic use of lithium, we believe that his renal insufficiency resulted from an overlooked link between lithium use and IgA-N with a possible genetic variation in SLC-T activity.

We refer back to Dr. Weder's study to understand the correlation of having a family history of HTN and the risk of lithium toxicity. Besides showing that the SLC-T activity and urinary lithium clearance are inversely related, he showed that normotensive patients with a family history of HTN had a significantly lower fractional lithium clearance than patients with no family history of HTN [12]. This further supports that having a family history might lower one's threshold for lithium toxicity. Plasma lithium concentrations can be promptly reduced by hemodialysis, but there is a widely recognized rebound increase in plasma lithium concentrations following dialysis [21]. Thus, it is necessary to routinely monitor Li levels, especially if the patient is at risk for lithium side effects and toxicity.

We summarize in Table 1 the pathological findings seen on renal biopsy among patients with IgA-N and in patients with lithium-induced tubulointerstitial nephritis [15, 19]. In Figure 2, we illustrate and propose a possible mechanism that results in lithium-induced nephropathy in IgA-N patients.

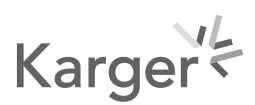


Case Reports

in Nephrology

and Dialysis

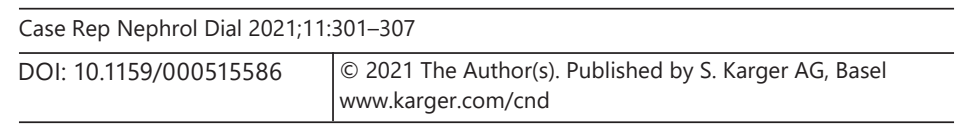

Mehandru et al.: IgA Nephropathy and Lithium Toxicity

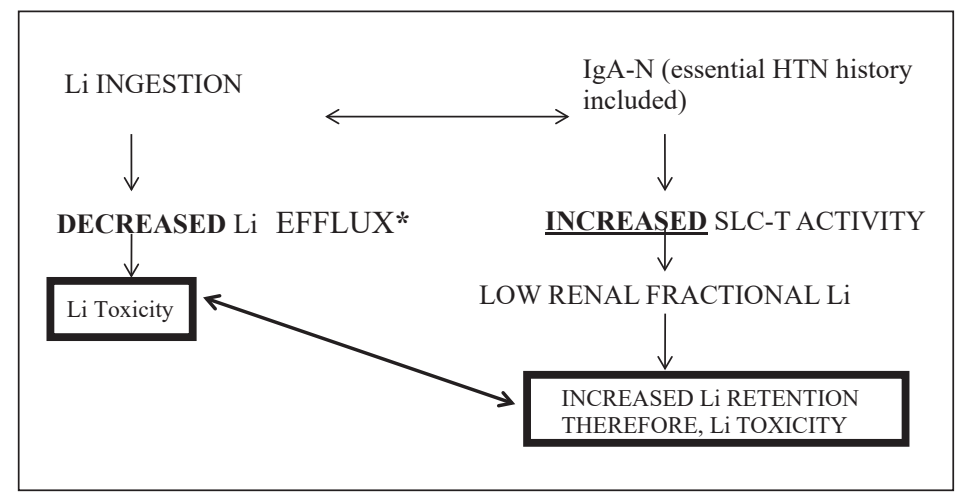

Fig. 2. Basic flowchart connecting the 2 pathological processes. SLC, sodium-lithium counter-transport; Li, lithium; HTN, hypertension; IgA-N, IgA nephropathy. *Note: decrease Li efflux accounts for the fact that all carriers are saturated, thus more risk of toxicity.

\section{Conclusion}

SLC-T is affected by lithium use, resulting in low Li efflux and, in turn, increasing the risk of lithium toxicity. Genetically susceptible disease processes, such as IgA-N where SLC-T expression is variable, further compromise lithium clearance. When treating bipolar disorders with lithium, clinicians must weigh all the possible risk factors that can increase serum lithium levels and possibly harm the patient. We recommend routine monitoring of lithium and serum creatinine levels in patients on lithium therapy and having comorbid conditions such as IgA-N, HTN, diabetes mellitus, or a family history of HTN. More studies are needed to clarify a complex interaction of lithium with SLC-T activity, especially in genetically variable diseases such as IgA-N and HTN. We highlight a unique case and recommend careful monitoring of lithium therapy in patients with IgA-N, HTN, and a family history of HTN.

\section{Statement of Ethics}

The authors have no statements to disclose for this publication.

\section{Conflict of Interest Statement}

The authors declare that there is no conflict of interests regarding the publication of this paper.

\section{Funding Sources}

The authors did not obtain funding for the manuscript.

\section{Author Contributions}

Sushil K. Mehandru: corresponding author: conceptualization and critical research. Supreet Kaur: literature review, drafting, and data collection. Aisha Ghias: literature review. Mohamed Bakr: data collection. Arif Asif: data analysis. Tushar J. Vachharajani: project administration. 


\section{Consent}

The patient described in the case report has given informed consent for the case report and accompanying images to be published. A copy of the written consent is available for review by the editor-in-chief of this journal.

\section{References}

1 Harvard Medical School. National Comorbidity Survey (NSC). 2007 [2017 Aug 21]. Data Table 2: 12-month prevalence DSM-IV/WMH-CIDI disorders by sex and cohort. Retrieved from: https://www.hcp.med.harvard. edu/ncs/index.php.

2 Ritchie H, Roser M. Mental health. 2018. Published online at OurWorldInData.org. Retrieved from: https:// ourworldindata.org/mental-health. [Online Resource]

3 Phelan KM, Mosholder AD, Lu S. A Lithium interaction with the cyclooxygenase 2 inhibitors rofecoxib and celecoxib and other nonsteroidal anti-inflammatory drugs. J Clin Psychiatry. 2003;64(11):1328.

4 Erden A, Karagöz H, Bașak M, Karahan S, Çetinkaya A, Avci D, et al. Lithium intoxication and nephrogenic diabetes insipidus: a case report and review of literature. Int J Gen Med. 2013;6:535-9.

5 Hedya SA, Avula A, Swoboda HD. Lithium toxicity. In: StatPearls [Internet]. Treasure Island, FL: StatPearls Publishing; 2020 Jan. [Updated 2020 Jul 19]. Available from: https://www.ncbi.nlm.nih.gov/books/ NBK499992.

6 West IC, Rutherford PA, Thomas TH. Sodium-lithium countertransport: physiology and function. J Hypertens. 1998;16(1):3-13.

7 Van Norren K, Thien T, Berden JH, Elving LD, De Pont JJ. Relevance of erythrocyte Na+/Li+ countertransport measurement in essential hypertension, hyperlipidaemia and diabetic nephropathy: a critical review. Eur J Clin Invest. 1998 May;28(5):339-52.

8 Lopes de Faria JM, Silveira LA, Morgano M, Pavin EJ. Erythrocyte sodium-lithium countertransport and proliferative diabetic retinopathy. Opthalmol Vis Sci. 2000 May;41(6):1482-5.

9 Ho KL, Rutherford PA, Thomas TH, Wilkinson R. Abnormal sodium-lithium countertransport kinetics in immunoglobulin A nephropathy patients and their families: association with hypertension. Am J Kidney Dis. 1996; 27(3):334-40.

10 Kontessis PS, Friedman R, Tariq T, Moro F, Williams DG, Hartley RB, et al. Sodium lithium coundertransport activity as a determinant of deterioration of glomerular function in IgA nephropathy. Exp Nephrol. 1994;2: 176-81.

11 Zheng X, Morrison AC, Turner ST, Ferrel RE. Association between SLC20A1 and sodium-lithium countertransport. Am J Hypertens. 2011;24:1069-72.

12 Weder AB. Red-cell lithium-sodium countertransport and renal lithium clearance in hypertension. N Engl J Med. 1986;314(4):198-201.

13 Canessa M, Adragna N, Solomon HS, Connolly TM, Tosteson DC. Increased sodium-lithium countertransport in red cells of patients with essential hypertension. N Engl J Med. 1980;302:772-6.

14 Simard M, Gumbiner B, Lee A, Lewis M, Norma D. Lithium carbonate intoxication. A case report and review of the literature. Arch Intern Med. 1989;149:36-46.

15 Emancipator SN. IgA nephropathy: morphologic expression and pathogenesis. Am J Kidney Dis. 1994;23(3): 451.

16 Boero R, Fabbri A, Esposti ED. Sodium-lithium countertransport activity in red blood cells of patients with IgA nephropathy. Am J Kidney Dis. 1993;21(5 Suppl 2):61-5.

17 Timmer RT, Sands JM. Lithium intoxication. J Am Soc Nephrol. 1999;10:666-74.

18 Ehrlich BE, Diamond JM. Lithium-induced changes in sodium-lithium countertransport. Biochem Pharmacol. 1981;30(18):2539-43.

19 Markowitz GS, Radhakrishnan J, Kambham N, Valeri AM, Hines WH, D’Agati VD, et al. Lithium nephrotoxicity: a progressive combined glomerular and tubulointerstitial nephropathy. J Am Soc Nephrol. 2000;11:1439-48.

20 Boero R, Degli Esposti E, Fabbri A, Guarena C, Forneris G, Quarello F, et al. Increased sodium-lithium countertransport activity in red cells of IgA nephropathy patients. Kidney Int. 1991 Dec;40(6):1118-22.

21 Scoble JE, McLean A, Munn S, Varghese Z, Sweny P, Moorhead JF, et al. Lithium nephrotoxicity and red cell lithium [letter]. Nephrol Dial Transplant. 1990;5(10):904. 